\title{
The interaction between fluid flow and ultra-hydrophobic surface in mini channel
}

\author{
Darina Jasikova ${ }^{1, *}$, Maja Gašič ${ }^{2}$, Simona Fialova ${ }^{3}$, Michal Kotek ${ }^{1}$ and Vaclav Kopecky ${ }^{1}$ \\ ${ }^{1}$ Department of Physical Measurement, Technical University of Liberec, The Institute for Nanomaterials, Advanced Technology and \\ Innovation, Studentska 1402/2 Liberec 1461 17, Czech Republic \\ ${ }^{2}$ Department of Physical Electronics, Faculty of Science, Masaryk University, Kotlarska 267/2, 61137 Brno, Czech Republic \\ ${ }^{3}$ Faculty of mechanical engineering, Brno University of Technology, Technicka 2896/0, Kralovo Pole, 61669, Brno, Czech Republic
}

\begin{abstract}
Interaction of liquid with ultra-hydrophobic surface is accompanied by creation of layer of air. The effect of the air film has a potential of use in industry in many applications. The quality of the surface is influenced by matrix roughness, the character of physical or chemical cover. There was developed a method for analysis of the liquid flow and the air film using the lighting in volume, visualization with CCD camera and long distance microscope, and optical filters. There were prepared four stainless steel samples of inner channel of dimensions $(80 \times 8 \times 8) \mathrm{mm}$ and initial surface roughness $\mathrm{Ra}$ 0.33, Ra 1.0, Ra 2.0, and Ra 2.2. The inner channel was treated with plasma and commercial hydrophobic coating Greblon (WEILBURGER Coatings $\mathrm{GmbH}$ ). There was realized study focused on the liquid flow velocity profile close to the air film. There are present results for laminar, transient and turbulent flows. The study also estimated the air film thickness depending on the Re number. The knowledge of the air film behaviour helps applied suitable degree of processing and cover for the target application.
\end{abstract}

\section{Introduction}

The ultra-hydrophobic surfaces have the prospect of great importance in industry, both in applications demanding easy cleaning, and they are presumed to reduce loss when the active parts of hydraulic machines are treated. Interaction of fluids with ultra-hydrophobic surface is accompanied by creation of layer of air, which depends on the quality of the surface. The quality of the surface is influenced by the matrix roughness, the character of physical or chemical cover. This properties lead to monolithic air layer presented as air film, or lead to plurality of bubbles of various sizes seated upon the surface.

The air layer can be observed visually at sufficient magnification and the dynamic interaction between fluid flow and air film can be studied with Global Imaging methods, particularly PIV. There is the velocity profile in the vicinity of the air layer in the main interest of the research. There was already described in the previous publication, focusing on the initial identification of an air layer, that the dynamics of the air layer is dependent on the velocity of the flowing liquid. This speed is expressed as Reynolds number, which is taken as a hydraulic diameter of the duct without an air layer. [1]

The initial measurement and identification of the air layer was performed on the testing device of hydraulic diameter $\mathrm{D}_{\mathrm{h}} 0.00375$ and extreme long entering distance - 80 times of the channel width to eliminate the effects of transient or underdeveloped flow pulsations. The test section was made of optically clear polycarbonate plastics with a defined roughness of interior walls. [1,2]

There was developed the a method for analysis of the flow and the air film using the lighting in volume, visualization with CCD camera and microscope lens, optical filters based on defined depth of field measuring plane. $[1,2]$

The next step was the experimental confirmation of theoretical assumptions about the nature of the air film on commercially used materials that have already been tested in hydraulic equipment - hydraulic pumps CKD Blansko. From industrial requirements resulted question, how is the air film depending on the roughness of the base material. There was a presumption of the smooth surface enabling more efficient and stable air film, but surface polishing increases production costs and it is not always consistently possible to all machined surfaces. There were fabricated four samples for testing purposes. These samples had defined inner duct's surface roughness corresponding to individual stages of material processing. The supposed result of measurement is to determine the nature of the air film depending on the Re number, the roughness of the material, and the character of the velocity field in the vicinity of the air film. [3]

Corresponding author: darina.jasikova@tul.cz 


\section{Experimental setup}

There were prepared four stainless steel samples of inner channel diameter $(80 \times 8 \times 8) \mathrm{mm}$. The inner part of the channel was machined to corresponding surface roughness. The sample surface marking followed ISO CSN 1302:1992 norm. The Ra 0.33 (average surface roughness $0.33 \mu \mathrm{m}$ ) corresponds to very fine edging and brushing, Ra 1.0 represent the surface finished with fine machining, such as turning, milling work or drilling. The rough surface represented with $\mathrm{Ra} 2.0$, and $\mathrm{Ra} 2.2$ substitute materials machined as prepared for finishing. The roughness of each sample was measured using standard laboratory device Mitutoyo Surftest SV-2000 after the machinery was finished.

The inner duct was further surface modified using plasma treatment to increase the contact angle. The open channels were covered with PCB optically clear cover and set with connection hoses. The samples were joined to the water circuit.

The system was enhanced with a linear precision voltage source KORADO KA3005P controllable from a PC and an adjustable voltage with an accuracy of hundredths.

The liquid - water was draw from the reservoir. The entrance length of the inlet straight duct was $1 \mathrm{~m}$ as well as the strait part on the outlet from the channel.

\subsection{Materials and Sample preparation}

Stainless steel samples $(80 \times 30 \times 15) \mathrm{mm}$ in size with commercial hydrophobic coating Greblon (WEILBURGER Coatings $\mathrm{GmbH}$ ) were used as a substrate. The experiment was carried out in the channels with dimensions of $(80 \times 8 \times 8) \mathrm{mm}$, which were placed in the middle of the sample. To obtain the super hydrophobic surface the samples were coated with super hydrophobic two-component (top and bottom) product Ultra Ever Dry developed by UltraTech international, Inc. Some samples (P-1L, P-2L) were pretreated by plasma to improve durability of super hydrophobic layer. In order to assess the plasma modification effect, the samples without plasma treatment were also prepared (R-1L, R-2L). The samples R-1L and P-1L are coated only with one of the Ultra Ever Dry components (top) to increase the layer homogeneity.

\subsubsection{Plasma pre-treatment and super hydrophobic layer preparation}

The atmospheric plasma slit-jet [reference] was used for samples pre-treatment. Electrodes were connected via an impedance matching unit to a radio frequency generator (Dressler) which is operated at a frequency of 13.56 MHz. The applied power was $300 \mathrm{~W}$. Argon (99.996\% Erproduct) was used as a working gas delivered at a flow rate of $50 \mathrm{ml} / \mathrm{s}$ into the plasma jet. The super hydrophobic product was subsequently applied according to the instructions using a commercial spray device. [4]

\subsubsection{Contact angle measurement}

Contact angle measurement was performed using SeeSystem 7.0 calculating the WCA on the basis of three-point interpolation of the drop height and width. Deionized water was used as a testing liquid and the volume of the drop was $2 \mu \mathrm{l}$.

Table 1. The sample marking, type of surface treatment, contact angle, and initial surface roughness.

\begin{tabular}{cccc}
\hline Sample & Treatment & Contact angle & $\begin{array}{c}\text { Surface } \\
\text { roughness }\end{array}$ \\
\hline R-1L & $\begin{array}{c}\text { Reference, } 1 \\
\text { component }\end{array}$ & $155 \pm 1$ & $0.33 \mu \mathrm{m}$ \\
\hline R-2L & $\begin{array}{l}\text { Reference, } 2 \\
\text { components }\end{array}$ & $153 \pm 1$ & $1 \mu \mathrm{m}$ \\
\hline P-1L & $\begin{array}{l}\text { Plasma pre- } \\
\text { treatment, } 1 \\
\text { component }\end{array}$ & $155 \pm 1$ & $2.0 \mu \mathrm{m}$ \\
\hline P-2L & $\begin{array}{l}\text { Plasma pre- } \\
\text { treatment, } 2 \\
\text { components }\end{array}$ & $154 \pm 1$ & $2.6 \mu \mathrm{m}$ \\
\hline
\end{tabular}

Prepared Samples:

ČK-KAN-A = R-2L Reference, 2 components

ČK-KAN-B $=$ P-2L Plasma pre-treatment, 2 components

ČK-KAN-C = R-1L Reference, 1 component

ČK-KAN-D = P-1L Plasma pre-treatment, 1 component

\subsection{Visualization techniques}

The visualization of the air film came out from the micro PIV measurement technique. This technique allows the visualization, tracking and evaluation processes within a very small scale of micrometres. The design of the measurement track and the samples were adapted to his method. The air film is shown as a light stripe that acts as lens. The air film refracts and reflects the illuminating light due its surface curvature. It also shows the mirroring effect. There is also perceptible surface structure in the picture. The effect of increasing flow rate is seen in Fig. 1.

The micro visualization technique provided us the information about the air film thickness. We used the long distance microscopy to cover the whole velocity profile across the channel $-5 \mathrm{~mm}$, and $8 \mathrm{~mm}$. The basis of micro PIV technique is a laser beam, which illuminates the entire volume of the examined space. For illumination of the evaluated area NewWave Gemini $\mathrm{Nd}$ :YAG pulsed laser is used, energy of $120 \mathrm{~mJ}$ per pulse length of $10 \mathrm{~ns}$, wavelength $532 \mathrm{~nm}$. The images are captured with Neo CMOS chip 5.5 MPixel size of 6.5 microns camera. Laser and camera system is controlled from a computer and synchronized with the external TimerBox.

Unlike standard PIV method, where the depth of field depends on the distance to the measurement object from the camera lens, f-number, in the micro PIV method depth of field is dependent on the parameters of the microscope lens, index of refraction of the liquid, and the wavelength of used light. Other parameters are 
related to the system of lenses: NA - iris, M - total magnification microscope, and $\mathrm{e}$ - the distance between the pixels on the CCD camera.

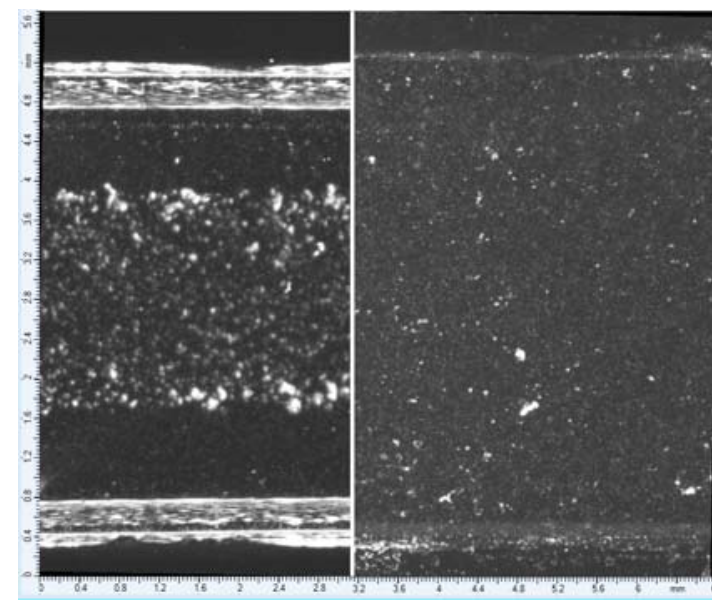

Fig. 1. View into the PCB channel: left side - air film upon the walls with the fluid flow Re 300 , right side - the same channel with the Re 3000 - clear walls without air film.

The arrangement of micro PIV has a constant depth of field $4 \mu \mathrm{m}$ at 10x magnification. This factor is important for choosing the size of seeding particles. Here in this investigating we used coated Rhodamine B $2 \mu \mathrm{m}$ fluorescent particles emitting light on $570 \mathrm{~nm}$. We used set of microscopic calibration targets for the calibration of the system to determine the magnitude coefficient.

The long distance microscopy method was used for the measurement of the velocity profile over the whole channel in one step. This optical setup was based on INFINIPROBETM TS-160 universal macro/micro imaging system that enables $4 \mathrm{x}$ and $16 \mathrm{x}$ magnification. This probe was working in the set of compressor for the wide field of view, and the working distance was $100 \mathrm{~mm}$ in the macro regime. The area of interest was illuminated with the laser light led with optical cable towards the sample.

The measurements run at $8 \mathrm{~Hz}$ frequency with 60 $\mathrm{mW}$ laser power at one flash. The light from the laser head was brought to investigated area with optical cable. For each measurement at least 290 records were always acquired and were further processed by the correlation function, validation and filtering before being evaluated statistics and estimated velocity profiles.

\section{Results and discussion}

The study of the velocity profile close to the air film, and the wall was based on the visualization using lightening in volume and the CCD camera with regulated shutter and defined depth of field. This setup allows us to visualize the thickness of the air film as well as the application of the cross-correlation analysing algorithms.

The entering distance was set according to the definition $L / D h / R e=C$, for $\mathrm{C}>0.03$ in the turbulent flow and $\mathrm{C}>0.08$ for the laminar flow. [2] We elongated the entering distance to avoid the flow instabilities up to $1 \mathrm{~m}$. The entering distance corresponds to 100times of channel widths.

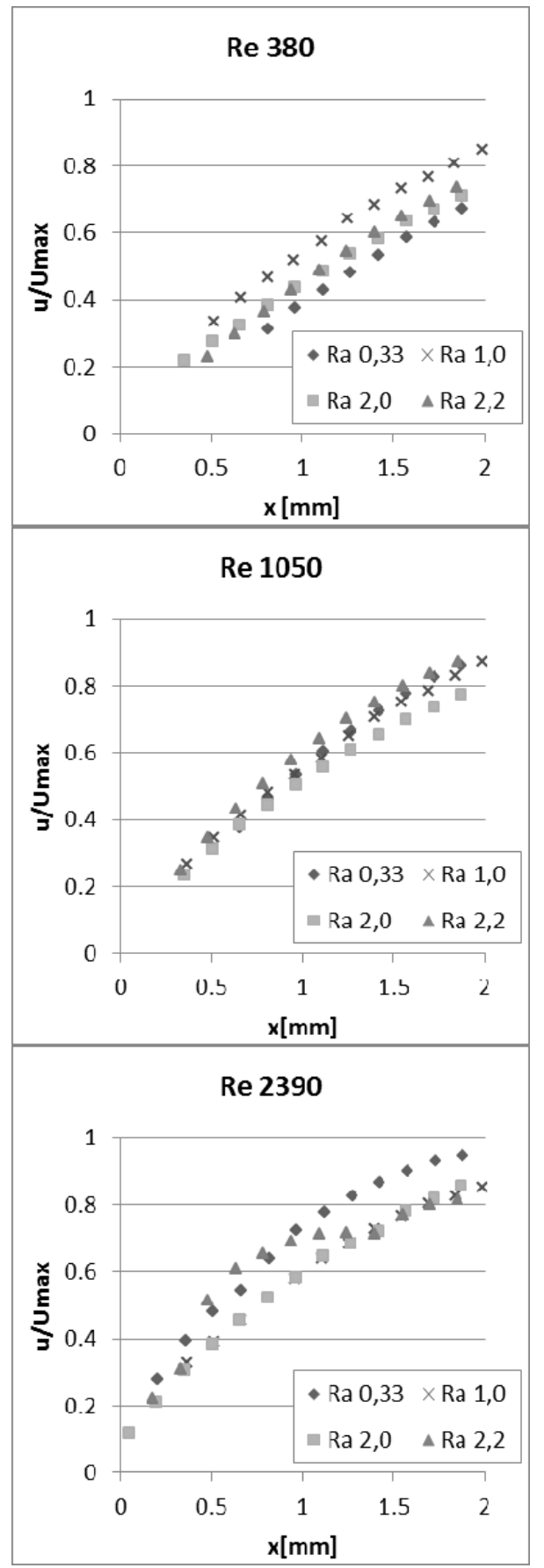

Fig. 2. The results of cross-correlation $(32 \times 32)$ px. The velocity profile normed to maximal velocity for the laminar flow Re 380, transient flow Re 1050 and the turbulent flow Re 2390. The $x[\mathrm{~mm}]$ axis represents the distance from the surface/channel's wall. Umax represents the maximal velocity in the channel.

The water channel was fulfilled with water avoiding the bubbles. The Reynolds number was fluently increased on selected values. Each liquid flow was stabilized at least 15 minutes. There was observed and captured the air film. There were tracked significant changes in air film thickness in time using $1 \mathrm{~Hz}$ sampling frequency. The air film was developed on each sample, 
but the quality of it varied depending on the surface roughness.

The thickness of air film on rough surface of Ra 2.0 and $\mathrm{Ra} 2.2$ corresponds to the air film created on PCB with $U E D \circledR$ nanoparticle. The average roughness of untreated surface was measured as $1.0 \mu \mathrm{m}$ (Ra 1.0). The samples treated with single layer of UED ${ }^{\circledR}$ nanoparticles increased in the roughness to $1.6 \mu \mathrm{m}$ ( $\mathrm{Ra} 1.6)$, and those were double treated increased in surface roughness from $2.4 \mu \mathrm{m}(\mathrm{Ra} 2.4)$. The global increase of the surface roughness can be explained due the UED ${ }^{\circledR}$ nanoparticles coagulation during the process. The sample ČK-KAN-C and ČK-KAN-D with the surface roughness Ra 2.0 and $\mathrm{Ra} 2.2$ are close those with particles coagulations.

The visualization was analysed using DantecStudio software. The images were processed to remove image noise, arithmetically analysed and masked. The processed images were cross-correlated using classical method at first. The resolution of the method corresponds to the camera resolution and magnifying lens system, i.e. after the calibration $1 \mathrm{px}$ is $4.7 \mu \mathrm{m}$ in the picture. The size of the interrogation area is $(32 \times 32) \mathrm{px}$. This means the size $(150.4 \times 150.4) \mu \mathrm{m}$ in the picture. The selected velocity profiles close to the air film are shown in Fig. 2.

Therefore, change in the position of first valid vector indicates a change in the size of the air layer to 151.4 $\mu \mathrm{m}$. This decrease corresponds to a change between Re of laminar flow, the critical flow, and the turbulent flow. If the initial thickness is smaller the shift in vector maps does not take effect. When selecting a smaller area we can no longer be sure of moving the particles within the evaluated areas, including appropriately chosen overlap.

The purpose of the analysis was the effect of air film on the velocity profile close to air layer. The other suitable method was the adaptive correlation. The setup of this analysing method was the initial interrogation area $(32 \times 32) \mathrm{px}$ and the final interrogation area $(16 \mathrm{x}$ 16) px with $0 \%$ overlap calculated in 3 iterations. The real size of the interrogation area was $(75.2 \times 75.2) \mu \mathrm{m}$ in this method so we got twice the resolution as in the first calculation. The changes in velocity profiles for selected Reynolds numbers are seen in figure 3.

There are changes in velocity profiles close to wall. We got higher resolution of results using adaptive correlation. This method uncovered tiny vortex structures that are developed in this stratified area of liquid flow. We also reached the calculation of first valid vector interrogation area closer to the wall, or air film. This effect is very well seen in Fig. $3-\mathrm{C}$ for Reynolds number 2390.

The initial velocity of the liquid flow was set repeatable for each sample and Reynolds number was calculation following the hydraulic diameter $D_{h} 0.008$. Anyway, the velocity profile was influenced of the air film that is unique for each sample, so the maximal velocity was not constant. We normed the velocity profile related to the maximal velocity on the central line. The effect of the air film is already described. [1] The velocity of liquid flow close to the air film is round coefficient $u_{x} / U_{\max } 0.3$ for the laminar flow. This coefficient responds to each maximal velocity on the central line of each channel. The velocity profiles are changed in the transient area of liquid velocities. There are makeable shift in position that are caused by the different position of the stratified flows. The profile shift is also caused by different maximal velocities. As it is seen in Fig. 3 the sample with Ra 2.0 and Ra 2.2 exhibits the same trend.

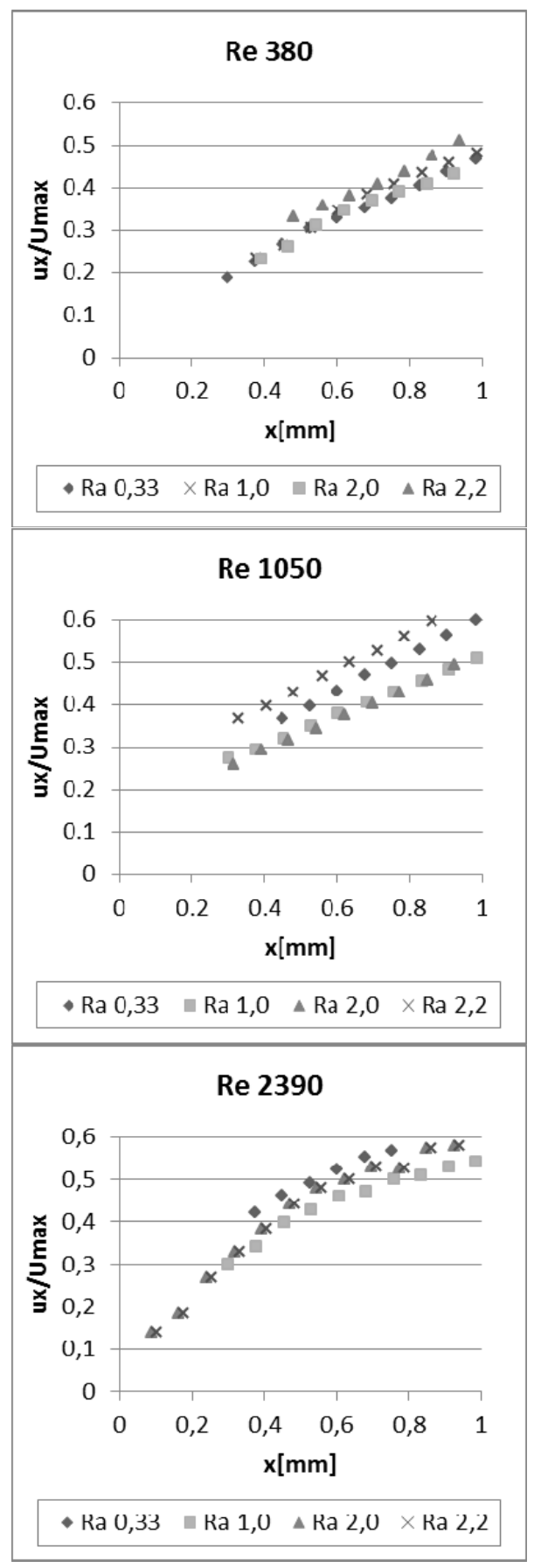

Fig. 3. The results of adaptive-correlation with initial interrogation area $(16 \times 16) \mathrm{px}$. The velocity profile normed to maximal velocity for the laminar flow Re 380 , transient flow Re 1050 and the turbulent flow Re 2390. The $x[\mathrm{~mm}]$ axis represents the distance from the surface/channel's wall. Umax represents the maximal velocity in the channel.

Markable difference is seen on turbulent flow. The border between the laminar flow and turbulent flow is in 
mini-channel shifted toward lower Reynolds number. Here we tried to reach the traditional edge of transient area that was limited by the pump. The velocity close to the disappearing air film is slowing down. Finally, the ratio between the local and maximal velocity decrease to 0.1 . The change in the velocity profile is set more positional than due the interaction with the air film.

There is seen in Fig. 3 for Re 2390 that the velocity profile for Ra 0.33 starts at $350 \mu \mathrm{m}$ far from the surface. The velocity of the initial point is only slightly higher comparing to the profiles without any air film. The maximal velocity on the central line is also higher as the channel cross-section is partly filled with air gap.

The second part of the research was dealing with the air film thickness estimation. The measurement of this property was based on image analysis of the intensity profiles over the selected line in the picture. Here we use the optical effect, that the air film reflect the light and can be visible as the light stripe. Due its geometrical character, it is rounded in the edges; it optically behaves as the lens. The air film is mirroring, and reflecting the emitted light from the seeding particles as well.

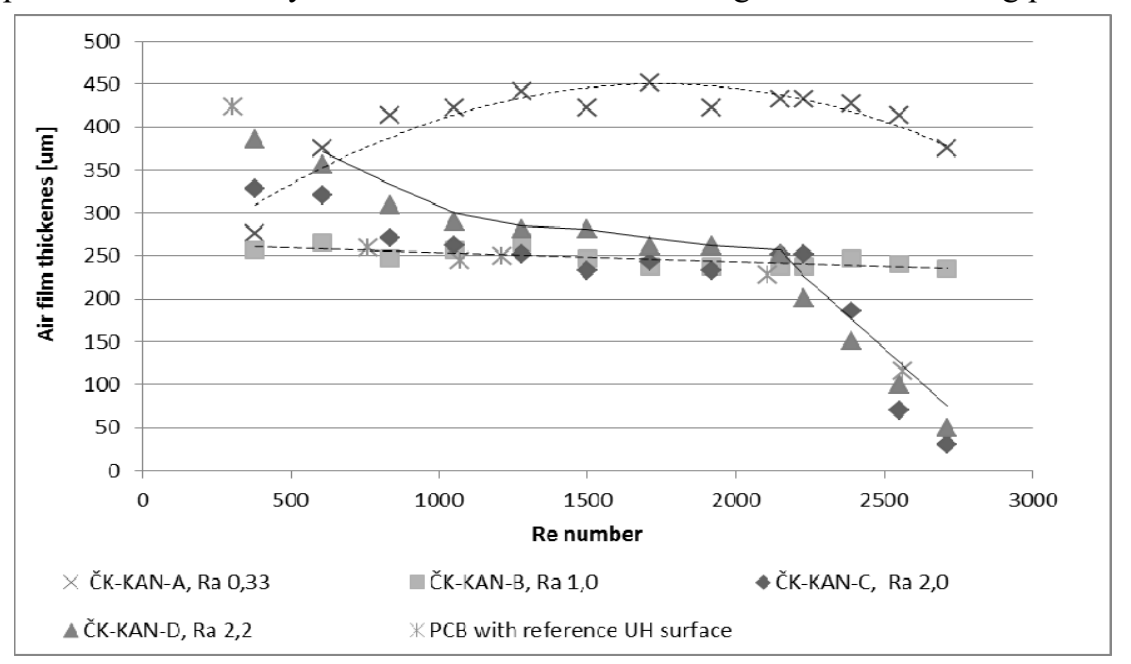

Fig. 4. The comparison of the air film thickness depending on the Reynolds number of the liquid flow for each sample. There is placed polycarbonate channel with UED ${ }^{\circledR}$ nanoparticles cover as a reference sample.

The difference in the intensities of pixels over the line is well determined. The thickness of the air film was calculated against the position of the surface. The values of air film was correlated to the Re number of liquid flow. The results are seen in the Fig. 4. The PCB sample covered with $\mathrm{UED}^{\circledR}$ nanoparticles is present as a reference sample. This sample has the surface roughness close to the stainless steel samples ČK-KAN-C with Ra 2.0, and $\check{C} K-K A N-D$ with Ra 2.2. This chart proves the dependence of the air film thickness on the surface roughness and it is not important, if the roughness is initial, ie. it is a material property, or it is developed due the covering process. This proof also corresponds with the theory of ultra-hydrophobicity on the rough defined surfaces. [5]

It is shown in Fig. 4 that the smooth surface of Ra 0.33 enables the air film existence over relatively high Re numbers. It was experimental prove the air film limitation due the liquid flow pulsation, flow instabilities, and Re numbers over 3000 . There is step change in the air film thickness for the Re number over this limit.

\section{Conclusions}

Here we presented the testing of materials that has great potential of usage in industry. There was not significant border between the surface roughness that is influenced by machinery processing, and the effect of the physical or chemical treatment. We tested the machined samples to investigate the dominant role of the surface background. Presenting graph negates doubts about the advantages of coarse structure to increase the degree of hydrophobicity of the material. This knowledge is crucial in design of dynamic hydraulic part of the system.

The results of this project LO1201 were obtained with cofunding from the Ministry of Education, Youth and Sports as part of targeted support from the "National Programme for Sustainability I " program and the support of TA03010950 "The use of hydrophobic and oleophobic surfaces during their interaction with liquids".

\section{References}

1. D. Jasikova, M. Kotek, S. Fialova, V. Kopecky, EPJ Web of Conferences, 114 (2016)

2. D. Jasikova, M. Kotek, V. Kopecky, AIP Conf. Proc., 1745 (2016)

3. S. Fialova, F. Pochyly, Wasserwirtschaft Extra, 1 (2015)

4. M. Klíma, etal., Method of generating plasma at atmospheric pressure in a slot jet and device for performance the method, WO2014094695A1, WO2014094695A4 (2014)

5. J. Bicoa, U. Thieleb, D. Quéréc, Colloids and Surfaces A: Physicochemical and Engineering Aspects, 206 (2002) 